\title{
Illumination control in lensless imaging for EUV mask inspection and review
}

\author{
Iacopo Mochi, Hyun-Su Kim, Uldis Locans, Atoosa Dejkameh, Ricarda Nebling, Dimitrios \\ Kazazis, and Yasin Ekinci \\ Paul Scherrer Institut, Villigen, Switzerland
}

\begin{abstract}
Coherence control and flexible pupil fill play a key role in the imaging of EUV reticles. This is also true for lensless metrology applications based on coherent diffraction imaging. We describe the concept and the key components of a Fourier synthesis illuminator designed to provide the RESCAN microscope with flexible illumination capabilities and to improve its resolution limit. In particular, we discuss the characteristics of the three mirrors of the new illuminator and the requirements for their multilayer coating.
\end{abstract}

Keywords: EUV mask review, inspection, defects, lensless imaging, coherent diffraction imaging, ptychography.

\section{INTRODUCTION}

As technological progress in the infrastructure for EUV lithography moves towards future technology nodes, the feature sizes present on photomask and wafers keep shrinking and the requirements for EUV metrology become more demanding. Even if commercial solutions for actinic pattern mask inspection (APMI) and EUV mask review are available, ${ }^{1,2}$ their cost of ownership remains relatively high and more development will likely be needed for future technology nodes.

In this scenario, we started exploring the possibility to carry out mask metrology with a lensless imaging approach based on coherent diffraction imaging (CDI). We developed the RESCAN microscope, a CDI-based EUV mask inspection platform operating at the Swiss Light Source synchrotron. In RESCAN, a sample is scanned with an EUV beam probe and a pixel detector collects the relative diffraction patterns. We then use ptychography to reconstruct the complex amplitude of the inspected sample and we compare it with an image of a reference area of the mask (die-to-die approach) or with an image calculated from the mask design (die-to-database approach) to identify the presence of defects.

We demonstrated lensless APMI with a defect sensitivity down to $50 \mathrm{~nm}$. We also demonstrated that RESCAN can be used on pellicle-protected masks and allows to detect defects on the pellicle surface as well. ${ }^{3}$

The current illumination system of RESCAN has a fixed chief ray angle of $6^{\circ}$ and a numerical aperture that can vary between 0.002 and 0.016 . This setting causes a fundamental limitation on the performance of the microscope. In a lensless system, the resolution limit depends on the acceptance angle of the detector while in a traditional imaging device, it is linked to the numerical aperture of the objective optics. To increase the resolution in RESCAN, it should be sufficient to increase the acceptance angle of the detector by choosing a larger detector or by reducing its distance from the sample. Unfortunately, the multilayer on the surface of the EUV mask is designed to reflect light at a wavelength of $13.5 \mathrm{~nm}$ with an incidence angle between $-12^{\circ}$ and $12^{\circ}$ and, beyond this angle range, the mask reflectivity drops rapidly. This limits the theoretical resolution of RESCAN to about $48 \mathrm{~nm}^{4}{ }^{4}$ To overcome this problem, we decided to upgrade our system with a Fourier synthesis illuminator ${ }^{5,6}$ to shift the portions of the diffraction spectrum that are normally unreachable, in the high-reflectivity angular range of the EUV mask. This approach will enable us to increase the resolution of RESCAN and will give it the flexibility to operate with arbitrary pupil shapes, paving the way towards EUV lensless mask review.

In this paper we review the characteristics of the Fourier synthesis illuminator we designed and we discuss the main challenges in the realization of its optical components.

Further author information: E-mail: iacopo.mochi@psi.ch 


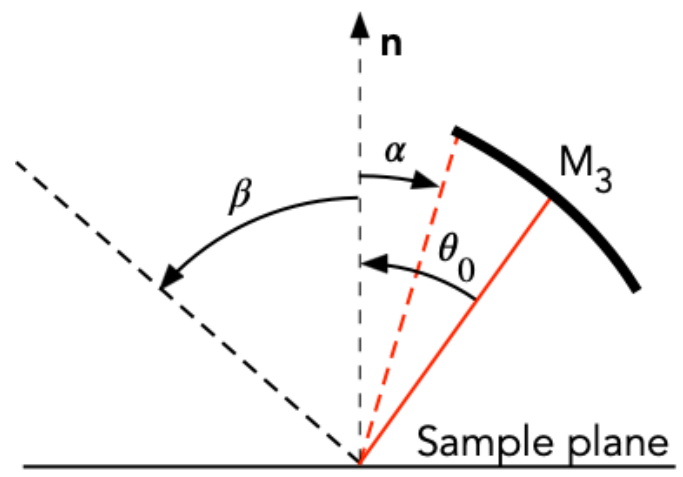

Figure 1. Diagram showing the chief ray angle $\theta_{0}$, the maximum angle allowed by the multilayer $\beta$, and the minimum angle of incidence allowed by the condenser mirror $\alpha$.

The parameters considered in the design of the illumination system are the single beam illumination NA, the probe size, shape, and intensity, the single-acquisition imaging NA, the maximum synthesizable imaging NA and the partial coherence of the single probe. To find a compromise between the optimal values of these parameters, we designed the three-mirror system shown in figure 2 .

\subsection{Probe size}

In the current configuration of RESCAN, the sample is close to the focal point of the condenser optics and our probe takes the shape of an Airy pattern. This approach maximizes the photon density over a circular area with a radius of 2 to $3 \mu \mathrm{m}$, depending on the condenser defocus and on the size of the aperture stop we use, giving high intensity high-order diffraction structures, but causing saturation and blooming in the low-order diffraction peaks on detectors with a limited dynamic range. This can be addressed by stitching multiple exposure together to create a high dynamic range image of the diffraction pattern, but it is a time consuming and error-prone procedure. For this reason, we decided to spread out the energy over a larger area by re-imaging an out-of-focus plane of the illumination beam onto the sample surface. The maximum size $D$ for the probe is limited by the coherence of the beam: ${ }^{7}$

$$
\frac{\lambda}{\Delta \lambda}>\frac{D}{\delta}
$$

where $\delta$ is the smallest feature that can be reconstructed, $\lambda$ is the wavelength and $\Delta \lambda$ is the illumination bandwidth. RESCAN illumination is provided by the XIL-II beamline which is equipped with a monochromator with $\lambda / \Delta \lambda=1500$ at $13.5 \mathrm{~nm}$. For a maximum achievable resolution limit of $20 \mathrm{~nm}$, the largest probe size would be $30 \mu \mathrm{m}$.

\subsection{Imaging NA}

The new system is designed to enable complex illumination patterns and provide coherence control for advanced imaging. This will come at the expense of a longer acquisition time because, to generate an arbitrary illumination pattern, it will be necessary to collect various datasets corresponding to the elements of the pupil that we want to synthesize. At the same time, it is important to preserve the ability of the microscope to perform fast, highcoherence illumination imaging with a single illumination probe. The imaging numerical aperture along the axis parallel to the incidence plane $\left(\mathrm{NA}_{Y}\right)$ is limited by the extent of the condenser optics as shown in figure $1 .{ }^{4}$ If $\alpha$ is the minimum incidence angle allowed by the condenser and $\beta$ is the maximum reflection angle allowed by the multilayer on the EUV mask sample, the maximum non vignetted $\mathrm{NA}_{Y}$ value for a chief ray angle $\theta_{0}$ is given by:

$$
\mathrm{NA}_{Y}=\min \left[\sin \left(\theta_{0}+\alpha\right), \sin \left(\beta-\theta_{0}\right)\right],
$$

and the maximum synthetic NA achievable is:

$$
\mathrm{NA}_{Y \max }=\sin (\beta-\alpha) .
$$

Assuming $\beta=14^{\circ}$, the current RESCAN configuration with a chief ray angle of illumination $\theta_{0}$ of $6^{\circ}$ and $\alpha=\theta_{0}$, has an effective $\mathrm{NA}_{Y}$ of 0.14 . In the new system $\alpha=3.5^{\circ}$ and the maximum synthetic $\mathrm{NA}_{Y \max }$ is 0.18 . 


\subsection{Illumination NA}

In the current RESCAN configuration, the illumination NA can be varied by selecting aperture stops with different diameters. With a circular stop with $1 \mathrm{~mm}$ diameter, the illumination NA is 0.004 . In the new design, with the same aperture size, we will have an illumination NA of 0.009 which translates into a reduced flux density on the detector and relaxes its dynamic range requirements. ${ }^{8}$

\begin{tabular}{|c|c|c|c|c|} 
& \multicolumn{2}{|c|}{ Current } & \multicolumn{2}{c|}{ New } \\
\hline Stop diameter $[\mathrm{mm}]$ & Probe size $[\mu \mathrm{m}]$ & NA & Probe size $[\mu \mathrm{m}]$ & NA \\
\hline 0.5 & 7.9 & 0.0021 & 5 & 0.0043 \\
1.0 & 3.9 & 0.0042 & 10 & 0.0087 \\
1.5 & 2.6 & 0.0062 & 15 & 0.0130 \\
2.0 & 2.0 & 0.0083 & 20 & 0.0174 \\
\hline
\end{tabular}

Table 1. Probe size as a function of the aperture stop diameter for the current and for the new optical configuration of RESCAN. In the current configuration, the condenser focuses the beam on the sample and the probe takes the shape of an Airy pattern; the probe size in this case is the Airy diameter. In the new configuration, the probe is an image of a defocused plane of the illumination beam and it has a circular shape.

\section{THE FOURIER SYNTHESIS ILLUMINATOR}

The current condenser of RESCAN will be substituted with the Fourier synthesis illuminator shown in figure 2. The new system will allow to synthesize larger and structured illumination pupils to extend the resolution of the microscope and to investigate the three dimensional properties of the EUV mask.

The EUV beam from the XIL-II beamline will be focused by a paraboloidal mirror $M_{1}$ and will be reflected by a tip-tilt mirror $M_{2}$ towards the condenser $M_{3}$. The points $P$ and $Q$ in the diagram of figure $2 \mathrm{~B}$ correspond to the foci of the ellipsoid to which the $M_{3}$ belongs. $P$ coincides with the pivot point of $M_{2}$ while $Q$ is located on the sample plane. The chief ray from the condenser mirror center $C$ to the focus $Q$, forms an angle of $6^{\circ}$ with the sample plane. By tilting $M_{2}$, it is possible to vary the chief ray angle of incidence on the sample surface without changing the position of the probe as shown in figure $2 \mathrm{~A}$.

A

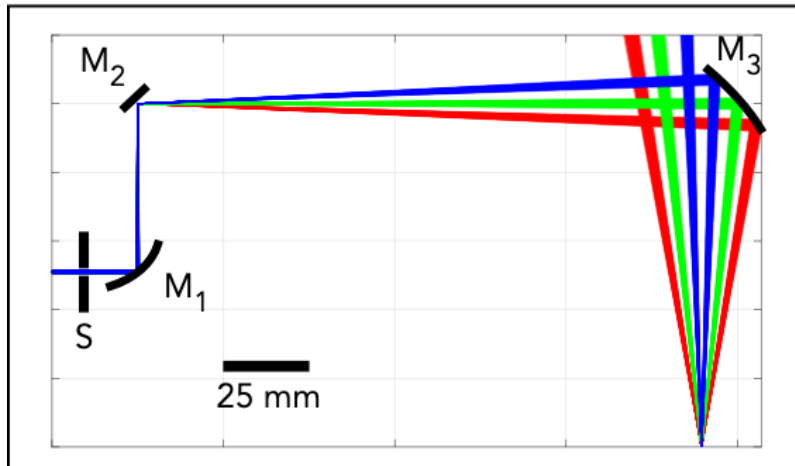

B

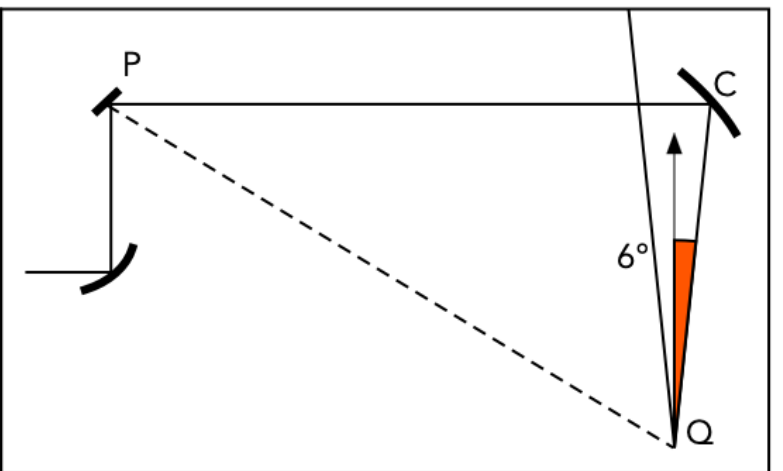

Figure 2. Optical layout of the Fourier synthesis illuminator. A. The EUV beam from the XIL-II beamline goes through a selectable aperture stop $(\mathrm{S})$ and is reflected vertically and focused by the paraboloidal mirror $M_{1}$ in proximity of the surface of a tip-tilt mirror $M_{2}$. The beam is then focused on the sample by the ellipsoidal condenser mirror $M_{3}$. B. The two foci of the ellipsoid to which $M_{3}$ belongs are $P$, the pivot point of $M_{2}$, and $Q$, a point on the sample surface. The ray from the center $C$ of $M_{3}$ to $Q$ forms an angle of $6^{\circ}$ with the normal to the sample plane.

The three mirrors that constitute the illuminator must be coated with a multilayer to ensure maximum reflection for EUV in the range of incidence angle they operate at. We calculated the angle of incidence distribution over the active surface of each of them and we verified that a suitable multilayer can be fabricated. 


\subsection{Paraboloidal mirror $\mathrm{M}_{1}$}

The XIL-II beamline produces a monochromatic EUV beam with a low divergence. With an aperture stop of $1 \mathrm{~mm}$ diameter, the beam divergence is $83 \times 47 \mu \mathrm{rad}^{2} . M_{1}$ is an off-axis paraboloidal mirror that reflects the light from the beamline at $90^{\circ}$ towards $M_{2}$ and focuses it $5 \mathrm{~mm}$ after the tip-tilt mirror surface. The aperture stop size and the focal length of $M_{1}$ determine the size of the beam footprint on $M_{2}$ and, as a consequence, the size of the probe on the sample. It is important to avoid to focus the beam on the surface of $M_{2}$ to limit the contamination, but at the same time, the beam footprint on $M_{2}$ can't be too large if we want to limit the size of the probe on the sample. The current design of the Fourier synthesis illuminator includes a single $M_{1}$ mirror with a focal length of $25 \mathrm{~mm}$, an off-axis distance of $\overline{C F}=50 \mathrm{~mm}$ and a diameter of $25 \mathrm{~mm}$ (figure 3A). The beam footprint on the mirror and its angle of incidence distribution is shown in figure 3B. Given the relatively simple shape of the mirror and its small size, it is possible to equip the new system with multiple instances of $M_{1}$ with different focal lengths to allow a more flexible control of the illumination properties.

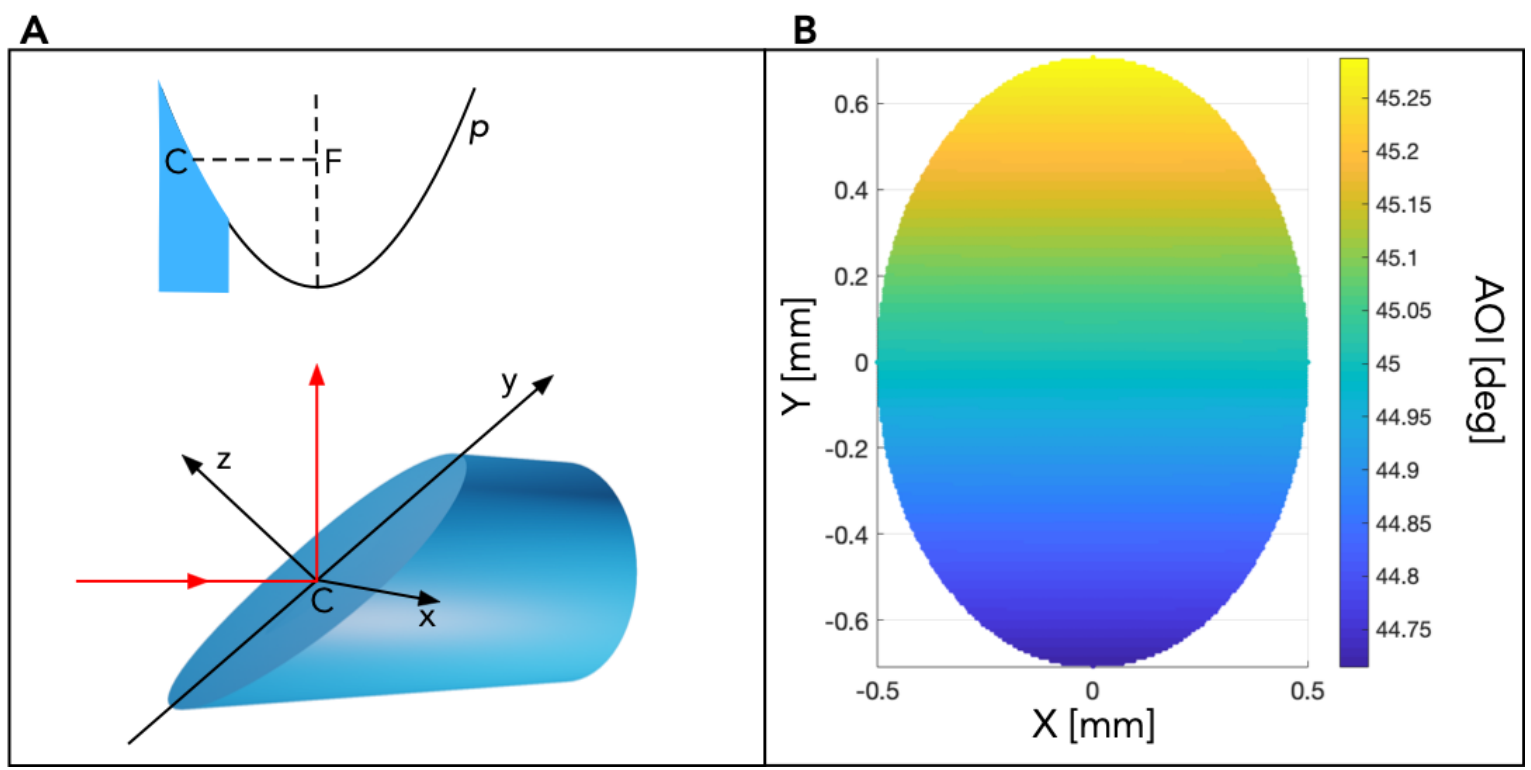

Figure 3. Angle of incidence on the surface of the $M_{1}$ mirror. A. Diagram of the geometry of $M_{1}$. The the $z$ axis of the reference frame used to describe the properties of the surface of the mirror coincides with the normal to the surface at its center. The chief ray is shown in red. B. Footprint of the beam on the surface of the mirror and angle of incidence distribution.

\subsection{Tip-tilt mirror $\mathrm{M}_{2}$}

The main concept of the Fourier synthesis illuminator is to control the angle of a pencil beam entering the illumination pupil. This functionality is provided by $M_{2}$, a flat tip-tilt mirror that reflects the beam focused by $M_{1}$ towards the condenser. $M_{2}$ will be mounted on a vacuum-compatible tip-tilt stage with a pivot point $P$ coinciding with the mirror surface. This condition will ensure that the probe, which is the image of the beam footprint on a plane containing $\mathrm{P}$ and forming an angle of $6^{\circ}$ with the chief ray (figure $2 \mathrm{~B}$ ), will remain stationary on the sample surface.

In its central configuration, $M_{2}$ forms an angle of $45^{\circ}$ with the incoming chief ray and it can rotate around a fixed pivot point $P$ located on its surface. The tip-tilt stage of the mirror has an angle range of $\pm 4^{\circ}$ around the $y$ axis and $\pm 3^{\circ}$ around the $x$ axis to cover the whole pupil defined by the condenser mirror $M_{3}$. This requirement implies that the the angle of incidence of the beam on $M_{2}$, showed in figure 4B for the central configuration, will actually go from $40^{\circ}$ to $48^{\circ}$, requiring an optimized broad-band multilayer coating.

The estimated EUV flux on the surface of $M_{2}$ is $10^{11}$ photons/s. To prevent carbon deposition on the mirror surface, the system will operate in ultra-high vacuum, or in a controlled $H_{2}$ atmosphere, but, as an additional 
measure, the mirror is designed with a rectangular shape in order to translate it laterally once the previously used area has become damaged or contaminated.

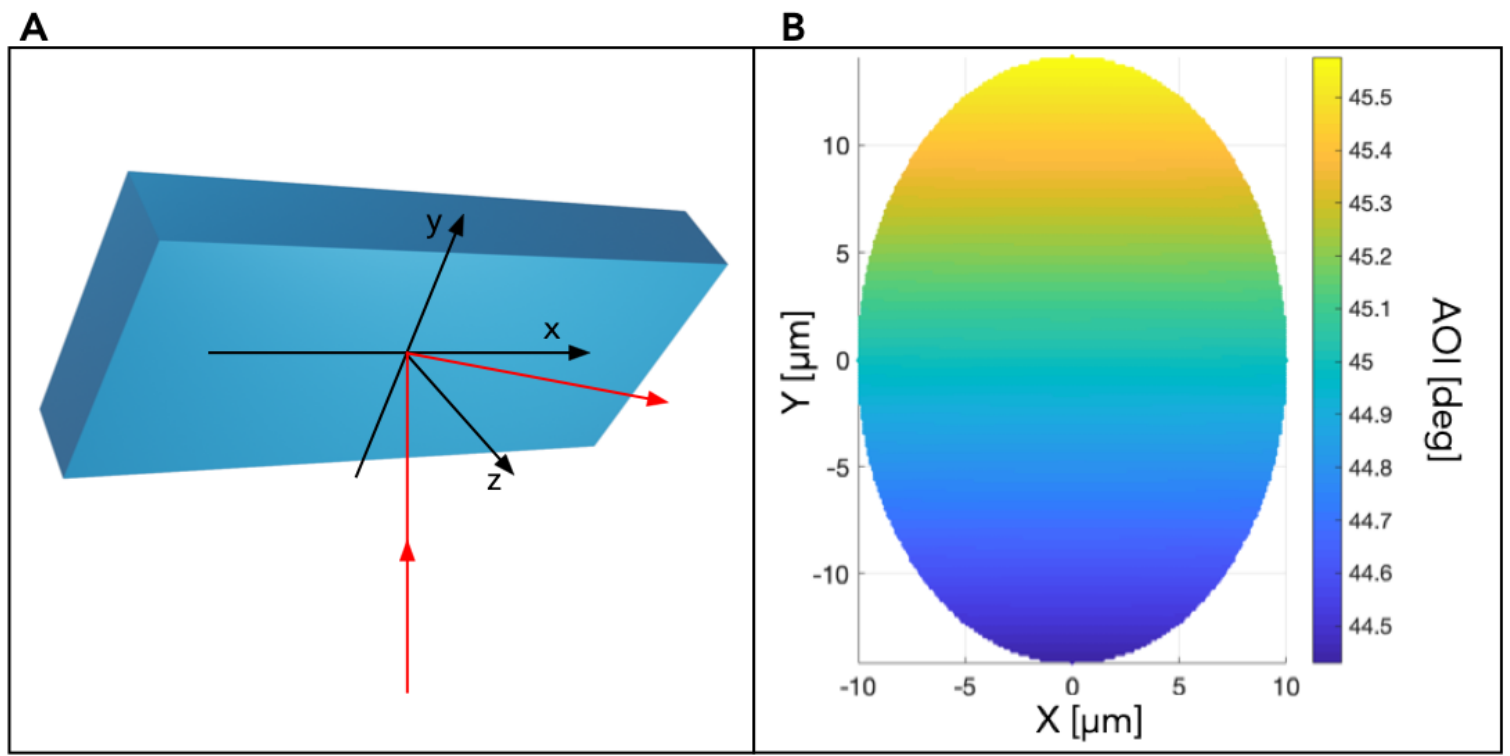

Figure 4. Angle of incidence on the surface of the $M_{2}$ mirror. A. Diagram of the geometry of $M_{2}$. The the $z$ axis of the reference frame used to describe the properties of the surface of the mirror coincides with the normal to the surface at its center. The chief ray is shown in red. B. Footprint of the beam on the surface of the mirror and angle of incidence distribution when $M_{2}$ is in its central configuration.

\subsection{Condenser mirror $\mathrm{M}_{3}$}

The beam steered by $M_{2}$ is reflected by $M_{3}$, an ellipsoidal condenser mirror with one focus located on $P$, the pivot point of the tip-tilt mirror, and the other on a point $Q$ on the sample surface as shown in figure $2 \mathrm{~B}$. The center $C$ of the condenser mirror is designed to reflect the chief ray so that $\overrightarrow{C Q}$ forms an angle of $6^{\circ}$ with the normal to the sample surface. The active area of the mirror, shown in figure $5 \mathrm{~B}$, has a keystone shape with a maximum extension of $27 \mathrm{~mm}$ in $y$ and $35 \mathrm{~mm}$ in $x$. The critical edge of this shape is the shorter arc at the bottom of the figure where the reflecting area must extend as much as possible towards the physical edge of the mirror to limit the vignetting of the light diffracted by the sample. A maximum edge of $1 \mathrm{~mm}$ can be tolerated. The footprint of the beam on $M_{3}$ is roughly elliptical with an extension of about $4 \mathrm{~mm}$ (figure $6 \mathrm{~A}$ ). The flux density on $M_{3}$ can be estimated as $6 \cdot 10^{10}$ photons $/\left(\mathrm{s} \cdot \mathrm{mm}^{2}\right)$. The magnification of $M_{3}$ changes slightly with the illumination angle and the diameter of the probe on the sample varies from 9 to $11 \mu \mathrm{m}$ for an aperture stop of $1 \mathrm{~mm}$ as shown in figure 6B. This is a not a problem for the image reconstruction algorithm employed in RESCAN because each illumination probe and its corresponding sample image is reconstructed independently. ${ }^{4}$

\subsection{Multilayer specifications}

The three mirrors of the illumination system work with different angles of incidence and they will need different multilayer designs to maximize their EUV reflectance. $M_{2}$ requires the largest range of incidence angles and for this reason, the design of its multilayer is the most critical. We verified the possibility to manufacture a suitable multilayer starting from a standard periodic $\mathrm{Mo} / \mathrm{Si}$ stack. The required angle range to optimize is $40^{\circ}$ to $48^{\circ}$, so we started with a periodic multilayer design with $40 \mathrm{Mo} / \mathrm{Si}$ bilayers and maximum reflectance centered at $44^{\circ}$. We optimized the thickness of each layer in order to minimize the merit function:

$$
f(\vec{t})=\int_{40^{\circ}}^{48^{\circ}}(R(\alpha, \vec{t})-T(\alpha))^{2} \mathrm{~d} \alpha,
$$


A

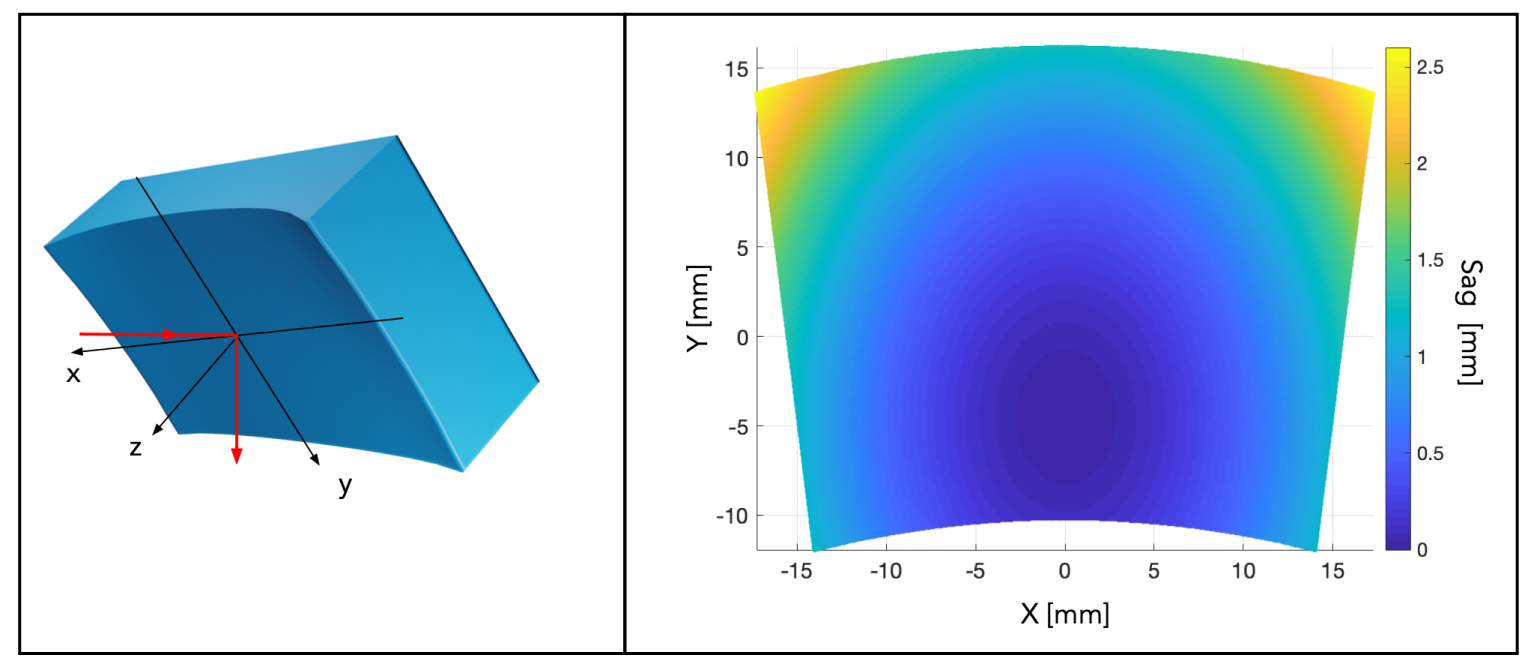

Figure 5. A. Diagram of the geometry of $M_{3}$. The $z$ axis of the reference frame used to describe the properties of the surface of the mirror coincides with the normal to the surface at its center. The chief ray is shown in red. B. Sag plot of the active area of the reflective surface of $M_{3}$.

A

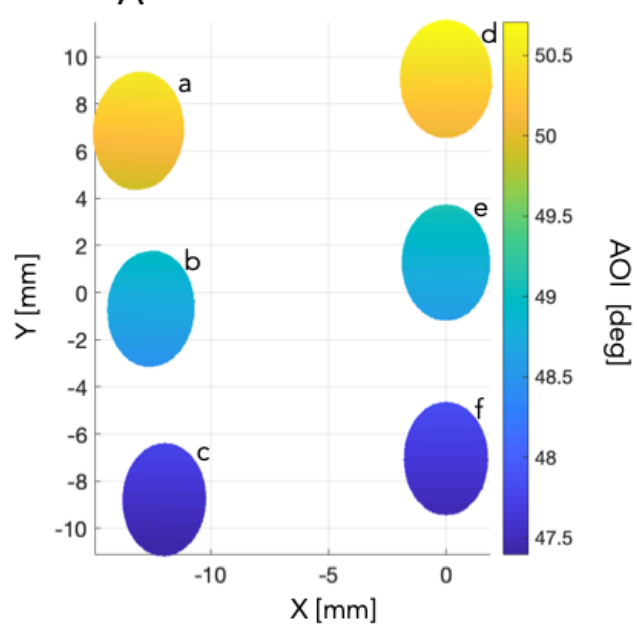

B
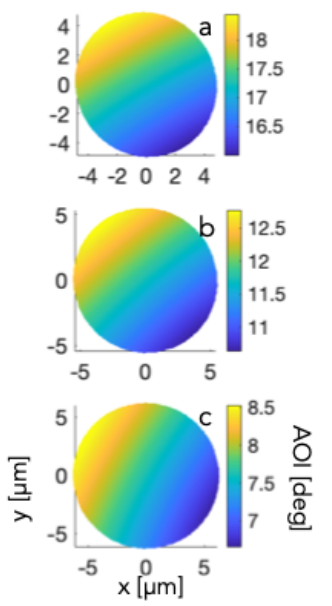
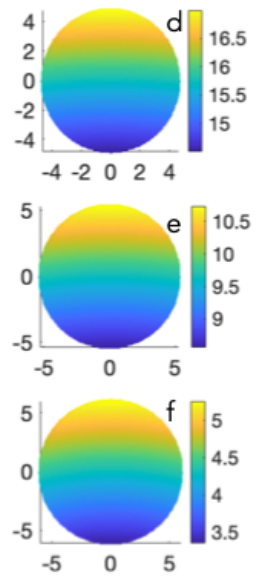

Figure 6. A. Position and angle of incidence of the beam on $M_{3}$ for six different angles of the tip tilt mirror. The reference frame used here is the same shown in figure 5A. B. Probe position and angle of incidence distribution on the sample surface. 


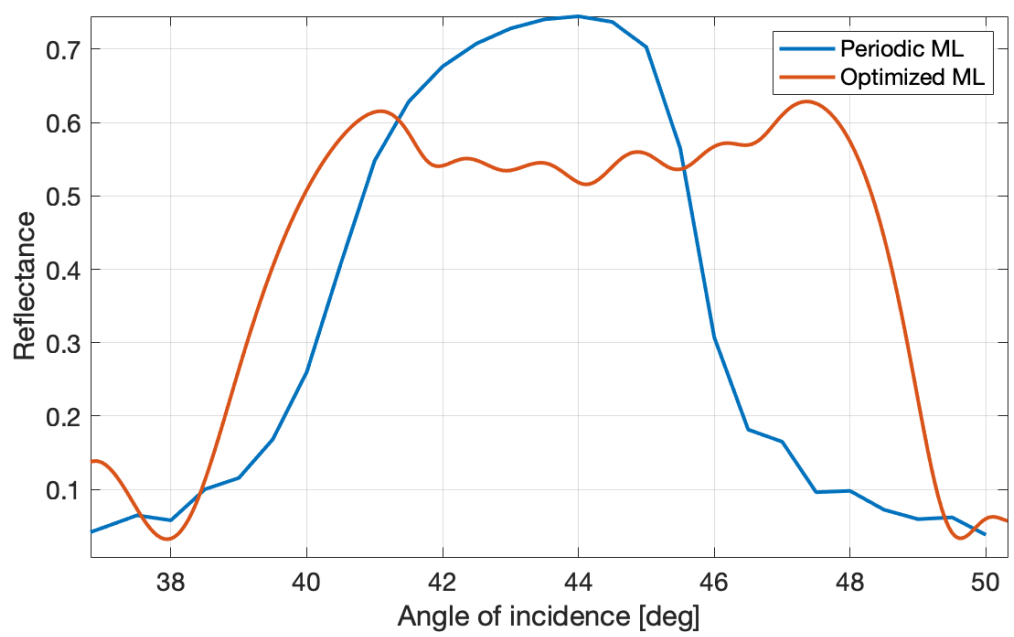

Figure 7. The blue curve represents the reflectance of a standard periodic Mo/Si multilayer with 40 bi-layers optimized for $13.5 \mathrm{~nm}$ and an angle of incidence of $44^{\circ}$. The red curve represents the reflectance of an a-periodic stack with the same number of layers optimized to cover a reflection angle range going from $40^{\circ}$ to $48^{\circ}$.

where $\vec{t}$ is a vector containing the thickness of each of the 80 layers, $R(\alpha, \vec{t})$ is the reflectance of the multilayer and $T(\alpha)$ is the target reflectance function. ${ }^{9}$ To calculate the multilayer reflectance, we developed a customizable MATLAB ${ }^{\circledR}$ function ${ }^{10}$ implementing a standard matrix multiplication method. ${ }^{11}$ Figure 7 shows that, optimizing the thicknesses of the 40 bilayers, we can obtain a large bandwidth multilayer with a reflectance above 0.5. This result could be further improved using more layers as shown in previous research. ${ }^{9,12}$

\section{OUTLOOK AND CONCLUSION}

We designed a new optical system to provide the RESCAN lensless microscope with flexible coherence control capability and to overcome the fundamental resolution limitations caused by the characteristics of the EUV mask multilayer. The system consists of a Fourier synthesis illuminator that will allow to change the illumination angle without displacing the probe on the sample. The illuminator has been developed with the goal to optimize several parameters of the microscope while keeping a flexible and affordable design to enable future upgrades when needed. The new illuminator, which will be implemented in the current RESCAN microscope, will be also a test bed for the potential development of a new lensless microscope for EUV mask metrology. This new microscope will be the evolution of the current RESCAN platform which was developed to demonstrate the potential of coherent diffraction imaging for EUV mask inspection. The concept is summarized in figure 8 and will include an $x, y$ reticle stage with interferometric position feedback, an optical microscope for pattern registration and navigation, and an automated mask loading system to avoid sample contamination. It will also feature an upgraded version of the Fourier synthesis illumination system described in this paper that will make it suitable for both mask inspection and review. With a maximum synthetic numerical aperture of 0.24 the system will have an imaging resolution of $28 \mathrm{~nm}$ on mask or $7 \mathrm{~nm}$ on wafer and will be able to provide reliable actinic mask metrology for the current and future technology nodes.

\section{ACKNOWLEDGMENTS}

The authors wish to thank Michaela Vockenhuber, Markus Kropf and José Gabadinho for their technical support for the measurements carried out at the SLS. This project has received funding from the Electronic Component Systems for European Leadership Joint Undertaking under grant agreement No 783247-TAPES3. This Joint Undertaking receives support from the European Union's Horizon 2020 research and innovation programme and Netherlands, Belgium, France, Germany, Israel. 


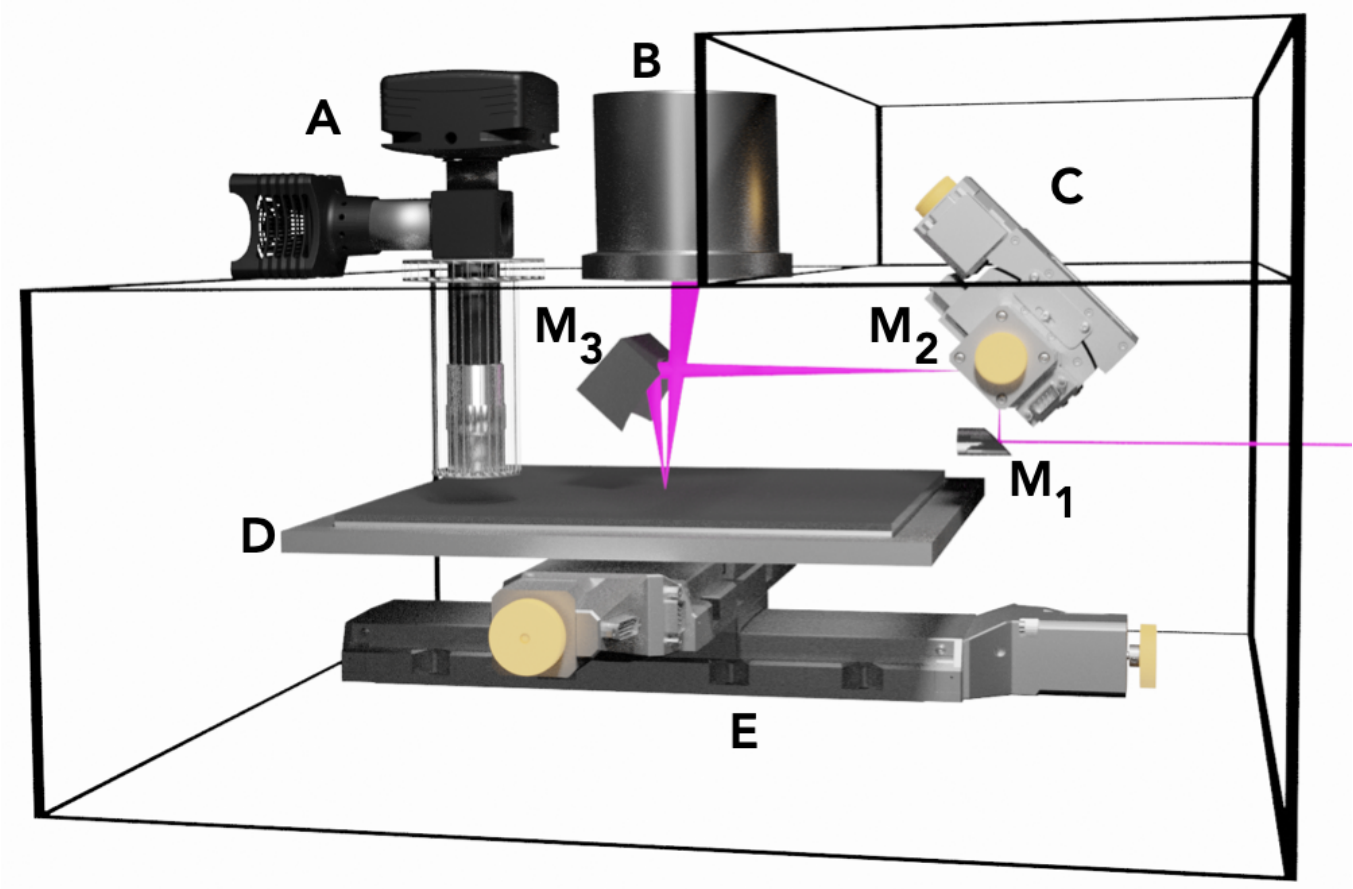

Figure 8. Conceptual rendering of a new, synchrotron-based lensless microscope for EUV mask inspection and review. A is a visible-light microscope for sample navigation, B is an EUV pixel detector, $\mathrm{C}$ is the tip-tilt stage for the $M_{2}$ mirror, $\mathrm{D}$ is the reticle holder, $\mathrm{E}$ is an $x, y$ stage with interferometric position feedback.

\section{REFERENCES}

[1] Capelli, R., Dietzel, M., Hellweg, D., Koch, M., Kersteen, G., Gwosch, K., and Pagel, D., "Actinic metrology platform for defect review and mask qualification: flexibility and performance," Proc.SPIE 10957 (2019). doi:10.1117/12.2518596.

[2] Miyai, H., Kohyama, T., Suzuki, T., Takehisa, K., and Kusunose, H., "Actinic patterned mask defect inspection for EUV lithography," in [Photomask Technology 2019], Rankin, J. H. and Preil, M. E., eds., Proc.SPIE 11148, 162 - 170 (2019). doi:10.1117/12.2538001.

[3] Mochi, I., Fernandez, S., Nebling, R., Locans, U., Helfenstein, P., Rajeev, R., Dejkameh, A., Kazazis, D., Tseng, L.-T., and Ekinci, Y., "Absorber and phase defect inspection on euv reticles using RESCAN," Proc.SPIE 10957 (2019). doi:10.1117/12.2515160.

[4] Mochi, I., Locans, U., Dejkameh, A., Nebling, R., Kazazis, D., Tseng, L.-T., and Ekinci, Y., "Resolution enhancement for lensless mask metrology with RESCAN," Proc.SPIE 11147, 156 - 163 (2019). doi:10.1117/12.2537068.

[5] Naulleau, P. P., Goldberg, K. A., Batson, P., Bokor, J., Denham, P., and Rekawa, S., "Fourier-synthesis custom-coherence illuminator for extreme ultraviolet microfield lithography," Appl. Opt. 42, 820-826 (Feb 2003).

[6] Mochi, I., Goldberg, K. A., Benk, M. P., and Naulleau, P. P., "Pupil shaping and coherence control in an EUV mask-imaging microscope," in [Photomask Technology 2013], Faure, T. B. and Ackmann, P. W., eds., Proc.SPIE 8880, 315 - 324 (2013).

[7] van der Veen, F. and Pfeiffer, F., "Coherent x-ray scattering," Journal of Physics: Condensed Matter 16, 5003-5030 (jul 2004).

[8] Williams, G. J., Quiney, H. M., Peele, A. G., and Nugent, K. A., "Fresnel coherent diffractive imaging: treatment and analysis of data," New Journal of Physics 12, 035020 (mar 2010). 
[9] Kuhlmann, T., Yulin, S. A., Feigl, T., and Kaiser, N., "EUV multilayer mirrors with tailored spectral reflectivity," in [X-Ray Mirrors, Crystals, and Multilayers II], Freund, A. K., Macrander, A. T., Ishikawa, T., and Wood, J. L., eds., Proc.SPIE 4782, 196 - 203 (2002). doi:10.1117/12.451348.

[10] Mochi, I., "Multilayer-mirror." https://zenodo.org/badge/latestdoi/207020995 (September 2019).

[11] Hecht, E., [Optics (4th Edition)], 426-428, Addison-Wesley (2001).

[12] Wang, Z., "Non-periodic multilayer coatings in EUV, soft x-ray and x-ray range," in [Advances in Optical Thin Films III], Kaiser, N., Lequime, M., and Macleod, H. A., eds., Proc.SPIE 7101, 373 - 387 (2008). doi:10.1117/12.797156. 